\title{
RETRACTION NOTE TO
}

\section{On-Chip Immunoassay for Molecular Analysis}

\author{
Andy Ng \\ (C) Springer International Publishing Switzerland 2016 \\ C.K. Dixit, A. Kaushik (eds.), Microfluidics for Biologists, \\ DOI 10.1007/978-3-319-40036-5_9
}

\section{Retraction Note:}

"On-Chip Immunoassay for Molecular Analysis," by Andy Ng, published as a chapter in Microfluidics for Biologists, edited by Chandra Dixit and Ajeet K. Kaushik, copyright 2016, ISBN 978-3-319-40035-8, is being retracted due to striking overlap with another published source, "Immunoassays in Microfluidic Systems", published 27 Apr 2010 in Analytical and Bioanalytical Chemistry volume 397 issue 3 on pages 991 to 1007 by Alphonsus H. C. Ng, Uvaraj Uddayasankar, and Aaron R. Wheeler.

The Editor(s) of this volume regret the oversight.

The updated online version of this chapter can be found under DOI 10.1007/978-3-319-40036-5_9 